for full evaluation of patients with impaired selectivity of protein excretion.

Most patients with severe glomerular abnormalities show additional features, particularly haematuria, hypertension, and impairment of renal function. ${ }^{112}$ Those with " membranoproliferative" glomerulonephritis may have reduced serum $B_{10}$-globulin levels. ${ }^{13}{ }^{14}$ Distinct patterns of immunoglobulin deposition can be detected by immunofluorescent techniques in glomeruli showing membranous and proliferative lesions. ${ }^{15}$ In short, all the diagnostic tests in use at present enable patients with steroid-resistant and progressive disease to be picked out with ease.

However, the diagnosis of "minimal change" does not necessarily imply complete recovery. From the mortality rates recorded in two recent follow-up studies of nephrotic children $^{16}{ }^{17}$ it seems unlikely that all or even most who died had initially severe glomerular lesions, in view of the frequency of minimal lesions in the children included in Cameron's report. The greatest problem, still unsolved, is how to distinguish children with minimal lesions who recover after one or two courses of corticosteroid therapy from those who respond but relapse repeatedly and require prolonged treatment. Many of the latter sooner or later develop signs of steroid toxicity, and since they may benefit from treatment with cyclophosphamide ${ }^{11} 1819$ it would be an advantage if they could be detected early. Unfortunately none of the available tests supply the answer, which at present can be discovered only by therapeutic trial.

Not all the patients included in the "minimal change" group have completely normal glomeruli and tubules. ${ }^{11}{ }^{14}$ Indeed, there is yet no entirely satisfactory definition of the term, and more detailed discussion of the morphological appearances and their significance is needed. Is it possible that minor differences in ultramicroscopic appearance, and seemingly trivial findings such as the presence of even transient hypertension or microscopical haematuria at onset, might bear a relation to the ultimate outcome ? Doubtless many of the patients being followed up by Cameron and his colleagues are being studied with these questions in mind, and a more detailed account in the future, giving clinicopathological correlations with the long-term response to treatment, will form a valuable addition to the present report.

\section{Psychogenic Vomiting}

Vomiting is often accompanied by few physical signs, and its multitude of causes may make diagnosis difficult. Not only is it extremely common, but it can also lead, whatever its cause, to dangerous dehydration and electrolyte deficiency. Added to this it may be a symptom of serious disease, such as a gastric carcinoma, renal or adrenal failure, or raised intracranial pressure.

Clinicians are rightly cautious in the handling of patients who complain of vomiting and are loath to make a diagnosis of a "functional" disorder. Most clinicians, however, recognize that there are some patients whose vomiting is related to psychological rather than organic causes and that these patients are perhaps more apt to produce vomiting as part of their symptom complexes than others. It has been less widely recognized that vomiting of psychological origin can be just as serious a symptom as its organic counterpart, and $\mathrm{O}$. W. Hill has previously noted ${ }^{1}$ the risks of loss of weight and potassium depletion with muscle paralysis that this type of vomiting carries. Proper detailed history-taking in patients with intractable vomiting will often show an obvious psychological cause, among which the most common are marital difficulties, often connected with sexual activity, and problems related to the health and habits of close relatives, such as alcoholism, loss of parental affection, and the problems of the difficult ageing relative. Hill found ${ }^{2}$ that in 12 of 20 patients with psychological vomiting there was an inescapable hostile relationship between the patient and his or her family group. Ten of the 20 also gave a history of recurrent vomiting in childhood, again often related to some major psychological trauma, such as a bereavement or admission to hospital or an orphanage.

The other interesting finding in this group of patients was the difference in the type of precipitating factor among these patients with psychogenic vomiting and others in whom abdominal pain was the major somatic symptom of psychiatric disease. In the latter group hostile relationships were less common, and they were outside rather than inside the family unit, so that it was possible for the patients to escape from the source of antipathy.

Hill points out that the one time when contact between close family members is often inescapable is at meal times. If hostility exists vomiting is a likely symptom, and it may appear to be related to food rather than the stressful situation of sitting and taking food with someone with whom the patient is in conflict. "It's enough to make you sick," one says, and indeed it may be. Clinicians would be well advised to remember this, since, though treatment of the underlying social situation may be impossible, symptoms improve with verbal catharsis and phenothiazine drugs.

\section{Where to Publish?}

Orthopaedic textbooks tell us that the Hippocratic method of reducing dislocations of the shoulder was in universal use until E. T. Kocher described ${ }^{1}$ his more logical method at the end of the nineteenth century. Kocher's method, however, had been described 3,000 years earlier.

About 1200 B.C. Rameses II had a tomb built at Thebes in the Nile Valley. The walls were painted by an artist called Ipuy, who broke with convention by using as his subject the building of the tomb. Among the incidents depicted are a workman gesticulating after an axe-head has fallen on to his foot, another having a foreign body removed from his eye, and a third whose dislocated shoulder is being reduced by Kocher's method. ${ }^{2}$ The patient is lying on the ground while the doctor (or osteopath) holds his arm, flexed at the elbow, with both hands-one at the elbow and the other at the wrist.

The moral of this episode is clear. Recognition of original work can be ensured only by publication in a reputable journal.

$$
\begin{aligned}
& \text { Kocher, E. T., Berl. klin. Wschr., 1870, 7, } 101 . \\
& 2 \text { Hussein, M. K., f. Bone ft Surg., 1968, 50B, } 669 .
\end{aligned}
$$

The next session of the General Medical Council will open on Tuesday, 26 November, at 2.15 p.m., when the President, Lord Cohen of Birkenhead, will take the chair and will deliver an address. 